\title{
PREVALENCE OF HYPOPIGMENTED MACULES
}

The prevalence of hypopigmented macules among 423 white individuals and their significance in the diagnosis of tuberous sclerosis (TS) were evaluated at the University of Washington School of Medicine and Children's Hospital, Seattle, WA. Twenty (4.7\%) had at least one macule, 4 of these had more than one macule, and none had more than three. Two (8\%) of 25 hypopigmented macules were identified only with a Wood lamp. Of the 20 individuals with macules, 13 had ophthalmoscopic exams and none showed retinal changes of tuberous sclerosis. A few hypopigmented macules on the skin of otherwise healthy individuals without a family history of TS do not warrant a search for other signs of the disorder. (Vanderhooft SL, Francis JS, Pagon RA, Smith LT, Sybert VP. Prevalence of hypopigmented macules in a healthy population. I Pediatr Sept 1996;129:355-61). (Reprints: Sheryll L Vanderhooft MD, University of Utah Health Sciences Center, Department of Dermatology, 50 North Medical Dr, Salt Lake City, UT 84132).

COMMENT. Hypopigmented macules are apparently more common than previously determined, occurring in close to $5 \%$ of the general population under 45 years of age. The presence of one to three macules is not by itself a significant risk factor for tuberous sclerosis. As a secondary feature of TS, at least one other manifestation must be present to establish a diagnosis.

\section{MYOCLONUS IN ANGELMAN SYNDROME}

A clinical and electroencephalographic study of 11 unrelated patients with Angelman syndrome (AS), confirmed by genetic analysis, is reported from the University of Pisa, Italy. All patients showed the jerky, tremulous, or dystonic motor pattern typical of AS. Using long-term video-EEG and polygraphic monitoring, these abnormal movements were shown to be a form of fast-bursting cortical myoclonus. Antimyoclonic treatment with piracetam in 5 patients produced a marked functional improvement. (Guerrini R, De Lorey TM, Bonanni P, et al. Cortical myoclonus in Angelman syndrome. Ann Neurol July 1996;40:39-48). (Respond: Dr Guerrini, Institute of Child Neurology and Psychiatry, University of Pisa, Via dei Giacinti 2, 56018 Calambrone, Pisa, Italy).

COMMENT. The diagnostic features of Angelman syndrome include ataxia, developmental delay, paroxysmal laughter, microcephaly, and seizures. The "puppetlike" movement disorder is related to a cortical myoclonus.

\section{TOXIC FACTORS IN ATTENTION DEFICIT}

\section{POLYCHLORINATED BIPHENYLS AND ATTENTION DEFICITS}

The effects of in utero exposure to polychlorinated biphenyls (PCBs) on cognitive function in 212 children at 11 years of age were tested at Wayne State University, Detroit, MI. Prenatal exposure to PCBs from maternal ingestion of contaminated Lake Michigan fish was associated with significantly lower full-scale and verbal IQ scores. Concentrations of PCBs in maternal serum and milk at delivery, only slightly higher than in the general population, caused long-term intellectual impairment, especially affecting memory, attention, and reading comprehension. (Jacobson JL, Jacobson SW. Intellectual impairment in children exposed to polychlorinated biphenyls in utero. N Eng I Med Sept 12 1996;335:783-9). (Reprints: Dr Joseph L Jacobson, Department of Psychology, Wayne State University, Detroit, MI 48202). 